Methodology Retrospective observational cohort study. We reviewed medical records of diagnosed EOC patients (pts) who were eligible for SACT, between 2012 and 2018. Primary endpoint was overall survival (OS). Secondary endpoints were description of platinum sensibility patterns and lines of treatment (LOT). Descriptive analysis of main demographic, clinical and treatment variables were performed. Kaplan-Meier method was used for OS. Uni and multivariable analysis were done using Cox proportional hazard analysis

Result(s)* We identified 268 EOC pts with median age of 66 (24-94). Debulking surgery was performed in 119 pts (44.4\%). Most were stage III-IV FIGO (200, 74.6\%) and had high-grade serous morphology (103, 38.6\%). BRCA mutations (germline and/or somatic) were detected in $7.6 \%$ of 131 tested pts. A third of pts never relapsed (86, 32.1\%). Platinum-based CT was the $1^{\text {st }}$ LOT in 173 pts (64,6\%). After relapse or progression, primary platinum resistance (PPR) was present in $34(19.7 \%)$, partial platinum sensibility in 29 $(16.8 \%)$ and full platinum sensibility in 24 (13.9\%). Of the 180 pts who progressed, $41(22.8 \%)$ were submitted to $2^{\text {nd }}$ SACT and $20(11.1 \%)$ to $3^{\text {rd }}$ SACT. Median number of LOT were $2(1-8)$. Bevacizumab concomitant with CT was used in 45 pts $(16.8 \%)$ at some point. PARPi was used in $23(8.6 \%)$ pts as maintenance treatment after $\geq 2$ platinum-based CT complete or partial response. Median OS was 25.5 months [IC95\% 19.55-35.42], which was significantly worse for more advanced disease [HR 8.46 IC95\% 4.13-17.31] and PPR [HR 2.72 IC95\% $1.63-4.54]$.

Conclusion* Our results confirm that EOC outcomes are modest and in line with other published cohorts. Multicentric realworld studies are needed to evaluate how innovative targeted therapies, recently introduced in the daily clinical practice, will change the course of this disease.

\section{PROGNOSTIC IMPACT OF MESENTERIC LYMPH NODE METASTASIS IN PATIENTS WITH ADVANCED OVARIAN CANCER}

CF Pop*, I Veys, A El Asmar, L Polastro, J Kerger, M Chintinne, D Larsimont, G Liberale. Institut Jules Bordet, Bruxelles, Belgium

\subsection{6/ijgc-2021-ESG0.473}

Introduction/Background* Digestive tract resection is a common procedure performed in bulky advanced-stage ovarian cancer (AOC) to achieve complete cytoreductive surgery (CRS). Very few data are available on the incidence and the prognostic impact of mesenteric lymph node (MLN) involvement.

The aim of this study is to determine the incidence of MLN involvement in patients undergoing a digestive tract resection at the time of CRS for AOC and to analyze its prognostic impact.

Methodology A retrospective study including patients who underwent CRS for AOC between 2006 and 2017. Patient's clinico-pathological characteristics were collected, incidence of various LN basin involvement were analyzed focusing on MLN. Overall survival (OS) and disease free survival (DFS) were calculated with the KM method.

Result(s)* During the study period 72 of 176 (40.9\%) patients with stage III-IVa AOC had a digestive tract resection during CRS with the recto-sigmoid resection being the most frequent (52 patients). There was an involvement of the serosa in 38
$(52.8 \%)$, sub-serosa in $10(13.9 \%)$, muscularis propria in 10 (13.9\%), and mucosa in 5 cases (6.9\%), respectively. MLN were found in 37 out of $72(51.4 \%)$ patients with digestive tract resection. 25 patients $(67.5 \%)$ had MLN involvement with a median of 5 involved nodes (range, 1 to 45). There was a trend in worse 5 -year OS $(45.8 \%$ vs $63.6 \%, \mathrm{p}=0.064)$ for patients with MLN involvement.

Conclusion* Patients with AOC involving the digestive tract is associated with a high incidence of MLN metastasis. Resection of the associated lymphatic tributaries of affected bowel segment and correct pathological examination of the MLN should be performed regularly to improve staging and better evaluate their prognostic impact in case of $\mathrm{LN}$ involvement.

\section{THE ROLE OF CA-125 AND HE-4 IN EPITHELIAL OVARIAN CANCER FOLLOW-UP}

J Castella*, Á Taus, B Esteban, S Espuelas, B Fabrego, E Miralpeix Rovira, JM Sole-Sedeno, $\mathrm{G}$ Mancebo. Spain

\subsection{6/ijgc-2021-ESGO.474}

Introduction/Background* Most patients diagnosed of advanced epithelial ovarian cancer (EOC) will relapse within the first years after the treatment. It is fundamental to find a marker for recurrence monitoring. Our objective was to perform a descriptive analysis of our data to estimate the rate of recurrence of EOC and the effectiveness of Ca-125 and HE-4 to agree with the radiologic diagnostic of relapse.

Methodology All patients were diagnosed of any stage of EOC from January 2014 to June 2020. They were retrospectively selected for the assessment of HE-4 and Ca-125 levels.

All patients selected had to undergo surgical treatment with optimal cytoreduction and chemotherapy if indicated, and they had to show radiological complete response after surgery. Out of 105 patients diagnosed during this period, 79 subjects met the inclusion criteria. 7 of them were lost during the followup, so 72 patients were included in the analysis.

Result(s) $* 47$ out of 72 patients relapsed during the follow-up $(65,3 \%)$. Out of all relapses, 32 patients had available data and reached normal levels of HE-4 after the surgery. 15/32 presented positive levels of HE-4 when the relapse was diagnosed (46,9\%). Moreover, in 6/32 cases the levels of HE-4 tend to increase in the moment of the relapse, even though the value did not become positive $(18,8 \%)$.

Out of all relapses, 43 patients had available data and reached normal levels of Ca-125 after the surgery. 20 out of 43 presented positive levels of $\mathrm{Ca}-125$ when the relapse was diagnosed (46,5\%). In addition, in $15 / 43$ cases the levels of Ca-125 tend to increase in the moment of the relapse, even though the value did not become positive (34,9\%).

30 patients had available data and reached normal levels of HE-4 and Ca-125 after the surgery. 9/30 cases presented positive levels of both HE-4 and Ca-125 in the moment of relapse $(30 \%)$.

Conclusion* Most patients showed an increase of biomarkers in agreement with radiologic relapse; in half of the cases the markers level became positive, but in some patients the values tend to increase despite being in the negative range. Both markers used together seem to be useful for the followup. 